\title{
激发发射荧光二阶校正算法快速检测桔子中噻苯 咪唑
}

\section{王雪梅，吴海龙”，聂瑾芳，李元娜，于永杰，俞汝勤} 湖南大学化学生物传感与计量学国家重点实验室, 长沙 410082

*联系人, E-mail: hlwu@hnu.cn

摘要 本文利用交替三线性分解(ATLD)和新提出的交替归一加权残差(ANWE)两种 二阶校正算法分别对三维激发发射荧光光谱数据进行解析，实现了对桔子中的噻苯咪 唑直接快速的定量测定. 当选取分析体系的组分数为 2 时, ATLD 和 AWNE 获得的平均 回收率分别为 $(99.7 \pm 3.3) \%$ 和 $(103.5 \pm 4.1) \%$. 另外, 还用栯圆置信区间测试(EJCR)和品质 因子，如灵敏度(SEN)、选择性(SEL)和检测下限(LOD)评估了两种算法的准确性. 实验 结果表明, 这两种算法能成功地用于直接分析桔子中噻苯咪唑的含量, 且 ANWE 的性

关键词

激发发射荧光光谱 交替三线性分解 交替归一加权残差 桔子提取液

噻苯咪唑

\section{1 引言}

噻苯咪唑(Thiabendazole, 简称 TBZ)具有杀菌性. 主要用于柑桔、苹果、香蕉等水果的保鲜. 而保鲜剂 用于果蔬采摘后，具有内吸性，可透过果皮进入果肉， 造成清洗不掉的残留. 残留的保鲜剂对人体肝、肾、 神经系统具有一定毒性 [1]. 为了确保消费者的安全, 各国制定了相应的法规和标准，规定了食品中保鲜 剂的最大残留量(http://postharvest.ifas.utl.edu). 欧盟、 日本、美国规定柑橘类水果中TBZ的用量不得超过 6 、 10、10 $\mathrm{mg} \cdot \mathrm{kg}^{-1}$, 我国限定用量为 $10 \mathrm{mg} \cdot \mathrm{kg}^{-1}$. 目前 用于食品中噻苯咪唑的测定方法有气相色谱-质谱法 [2]、高效液相色谱-紫外检测法 ${ }^{[3]}$ 、高效液相色谱-质谱

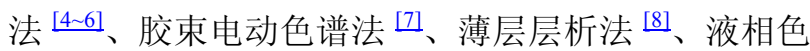
谱-电喷雾飞行时间质谱法和液相色谱-离子阱质谱 法 [9]等. 也有应用噻苯咪唑具有内源荧光特性而测其
含量的报道, 如反相高效液相色谱-荧光检测法 $[10]$ 、 多通道荧光光学传感器法 ${ }^{[11]}$, 还有利用其在碱性条 件下溶于乙酸乙酯，而在酸性条件下溶于水中的特 性将蒜苔中干扰物去除，然后用菼光分光光度法测 定 ${ }^{[12]}$ 的报道. 这些方法存在预处理复杂，费用高和 使用大量有毒溶剂等不足. 若引入化学计量学方法, 以“数学分离”替代“化学分离”, 则能实现复杂成分的 直接快速测定．曾有用平行因子分析算法结合三维 荧光光谱法对多菌灵、呋喃基苯并咪唑、噻苯咪唑组 成体系进行分辨 ${ }^{[13]}$ ，但未见用三维荧光二阶校正法 测定其实际背景中的噻苯咪唑含量的报道. 本文采 用交替三线性分解算法(ATLD) $)^{[14]}$ 及新提出的交替归 一加权残差算法(ANWE) ${ }^{[15]}$ 分别与三维荧光光谱相 结合, 对桔子中噻苯咪唑进行了直接定量分析, 方法 简单、快速，且具有未知干扰共存也不影响其感兴趣 成分定量分析结果的优点. 


\section{2 方法原理}

\section{1 三线性成分模型}

将 $K$ 个样本在选取激发波长数为 $I$ 、发射波长数 为 $J$ 条件下进行测定, 得到的激发发射光谱矩阵可构 成三维响应数阵 $\underline{\mathbf{X}}(I \times J \times K)$, 它满足下面这个三线 性成分模型:

$$
\begin{gathered}
x_{i j k}=\sum_{n=1}^{N} a_{i n} b_{j n} c_{k n}+e_{i j k}(i=1,2, \cdots, I ; \\
j=1,2, \cdots, J ; k=1,2, \cdots, K)
\end{gathered}
$$

其中 $N$ 表示对体系有实际贡献的荧光成分数; $x_{i j k}$ 是三 维响应数阵 $\underline{\mathbf{X}}$ 中的元素 $(i, j, k)$, 它表示第 $k$ 个样本在 激发波长为 $i$ 、发射波长为 $j$ 时的苂光强度; $c_{k n}$ 是相对 浓度阵 $\mathbf{C}(K \times N)$ 中的元素 $(k, n) ; a_{i n}$ 是相对激发光谱 阵 $\mathbf{A}(I \times N)$ 中的元素 $(i, n) ; b_{j n}$ 是相对发射光谱阵 $\mathbf{B}(J$ $\times N)$ 中的元素 $(j, n) ; e_{i j k}$ 是三维残差数阵 $\underline{\mathbf{E}}(I \times J \times K)$ 中的元素 $(i, j, k)$.

\section{2 交替三线性分解(ATLD)算法 ${ }^{[14]}$}

通过利用交替最小二乘原理，借助基于奇异值 分解(SVD)的 Moore-Penrose 广义逆计算和交替迭代 步骤来改进三线性分解的性能，使损失函数即残差 阵元素的平方总和达到极小化. 该算法可同时获得 关于三维数阵 $\underline{X}$ 的相对激发光谱阵 $\mathbf{A}$ 和相对发射光 谱阵 B 以及相对浓度阵 C. 对 A、B、C 作适当后处 理，再根据文献[14]的二阶校正步骤作未知样中感兴 趣组分的直接定量分析.

\section{3 交替归一加权残差(ANWE)算法}

ANWE算法为本室近年来新提出的三维数阵分 解算法 ${ }^{[15]}$, 它通过最小化以下三个目标函数来分解 模型:

$$
\begin{aligned}
\mathrm{S}(\mathbf{C})= & \sum_{i=1}^{I}\left\|\mathrm{D}_{\mathrm{B}} \mathbf{B}^{+}\left(\mathbf{X}_{i . .}-\mathbf{B} \operatorname{diag}\left(\mathbf{a}_{(i)}\right) \mathbf{C}^{\mathrm{T}}\right)\right\|_{\mathrm{F}}^{2} \\
& +\sum_{j=1}^{J}\left\|\mathrm{D}_{\mathrm{A}} \mathbf{A}^{+}\left(\mathbf{X}_{j .}-\mathbf{C d i a g}\left(\mathbf{b}_{(j)}\right) \mathbf{A}^{\mathrm{T}}\right)^{\mathrm{T}}\right\|_{\mathrm{F}}{ }^{2}, \\
\mathrm{~S}(\mathbf{A})= & \sum_{j=1}^{J}\left\|\mathrm{D}_{\mathrm{C}} \mathbf{C}^{+}\left(\mathbf{X}_{. j .}-\mathbf{C d i a g}\left(\mathbf{b}_{(j)}\right) \mathbf{A}^{\mathrm{T}}\right)\right\|_{\mathrm{F}}^{2} \\
& +\sum_{k=1}^{K}\left\|\mathrm{D}_{\mathrm{B}} \mathbf{B}^{+}\left(\mathbf{X}_{. . k}-\mathbf{A d i a g}\left(\mathbf{c}_{(k)}\right) \mathbf{B}^{\mathrm{T}}\right)^{\mathrm{T}}\right\|_{\mathrm{F}}{ }^{2},
\end{aligned}
$$

$$
\begin{aligned}
\mathbf{S}(\mathbf{B})= & \sum_{k=1}^{K}\left\|\mathrm{D}_{\mathrm{A}} \mathbf{A}^{+}\left(\mathbf{X}_{. . k}-\mathbf{A d i a g}\left(\mathbf{c}_{(k)}\right) \mathbf{B}^{\mathrm{T}}\right)\right\|_{\mathrm{F}}^{2} \\
& +\sum_{i=1}^{I}\left\|\mathrm{D}_{\mathrm{C}} \mathbf{C}^{+}\left(\mathbf{X}_{i . .}-\mathbf{B} \operatorname{diag}\left(\mathbf{a}_{(i)}\right) \mathbf{C}^{\mathrm{T}}\right)^{\mathrm{T}}\right\|_{\mathrm{F}}{ }^{2},
\end{aligned}
$$

这里,

$$
\begin{aligned}
& \mathrm{D}_{\mathrm{A}}=\operatorname{diag}\left(\operatorname{sqrt}\left(\mathbf{1} . / \operatorname{diagm}\left(\mathbf{A}^{+}\left(\mathbf{A}^{\mathrm{T}}\right)^{+}\right)\right)\right), \\
& \mathrm{D}_{\mathrm{B}}=\operatorname{diag}\left(\operatorname{sqrt}\left(\mathbf{1} . / \operatorname{diagm}\left(\mathbf{B}^{+}\left(\mathbf{B}^{\mathrm{T}}\right)^{+}\right)\right)\right), \\
& \mathrm{D}_{\mathrm{C}}=\operatorname{diag}\left(\operatorname{sqrt}\left(\mathbf{1} . / \operatorname{diagm}\left(\mathbf{C}^{+}\left(\mathbf{C}^{\mathrm{T}}\right)^{+}\right)\right)\right) .
\end{aligned}
$$

令 $\mathrm{W}_{\mathrm{A}}=\mathrm{D}_{\mathrm{A}} \mathbf{A}^{+}, \mathrm{W}_{\mathrm{B}}=\mathrm{D}_{\mathrm{B}} \mathbf{B}^{+}$和 $\mathrm{W}_{\mathrm{C}}=\mathrm{D}_{\mathrm{C}} \mathbf{C}^{+}$, 可看作归一化 加权因子. 根据以上提出的目标函数, 用交替归一加 权残差法进行分解. 由于通常情况下, A、B 和 C 满足 列满秩，所以在以交替的方式最小化 ANWE 残差时, 方程(2)中固定 A、B 后获得的新的 C 为

$$
\begin{aligned}
\mathbf{C}= & {\left[\sum_{i=1}^{I} \mathbf{X}_{i . .}{ }^{\mathrm{T}}\left(\mathbf{B}^{+}\right)^{\mathrm{T}} \mathbf{D}_{\mathrm{B}}{ }^{2} \operatorname{diag}\left(\mathrm{a}_{(i)}\right)\right.} \\
& \left.+\sum_{j=1}^{J} \mathrm{X}_{. j .}\left(\mathbf{A}^{\mathrm{T}}\right)^{+} \mathbf{D}_{\mathrm{A}}{ }^{2} \operatorname{diag}\left(\mathrm{b}_{(j)}\right)\right] \\
& \times\left[\sum_{i=1}^{I}\left(\mathbf{D}_{\mathrm{B}} \operatorname{diag}\left(\mathrm{a}_{(i)}\right)\right)^{2}+\sum_{j=1}^{J}\left(\mathbf{D}_{\mathrm{A}} \operatorname{diag}\left(\mathrm{b}_{(j)}\right)\right)^{2}\right]^{+} .
\end{aligned}
$$

方程(3)中固定 $\mathbf{C} 、 \mathbf{B}$, 获得新的 $\mathbf{A}$ 为:

$$
\begin{aligned}
\mathbf{A}= & {\left[\sum_{j=1}^{J} \mathbf{X}_{. j .}{ }^{\mathrm{T}}\left(\mathrm{C}^{+}\right)^{\mathrm{T}} \mathbf{D}_{\mathrm{C}}{ }^{2} \operatorname{diag}\left(\mathrm{b}_{(j)}\right)\right.} \\
& \left.+\sum_{k=1}^{K} \mathbf{X}_{. . k}\left(\mathbf{B}^{\mathrm{T}}\right)^{+} \mathbf{D}_{\mathrm{B}}{ }^{2} \operatorname{diag}\left(\mathrm{c}_{(k)}\right)\right] \\
& \times\left[\sum_{j=1}^{J}\left(\mathbf{D}_{\mathrm{C}} \operatorname{diag}\left(\mathrm{b}_{(j)}\right)\right)^{2}+\sum_{k=1}^{K}\left(\mathbf{D}_{\mathrm{B}} \operatorname{diag}\left(\mathrm{c}_{(k)}\right)\right)^{2}\right]^{+} .
\end{aligned}
$$

方程(4)中固定 $\mathbf{A} 、 \mathbf{C}$, 获得新的 $\mathbf{B}$ 为:

$$
\begin{aligned}
\mathbf{B}= & {\left[\sum_{k=1}^{K} \mathrm{X}_{. . k}{ }^{\mathrm{T}}\left(\mathbf{A}^{+}\right)^{\mathrm{T}} \mathbf{D}_{\mathrm{A}}{ }^{2} \operatorname{diag}\left(\mathrm{c}_{(k)}\right)\right.} \\
& \left.+\sum_{i=1}^{I} \mathrm{X}_{i . .}\left(\mathrm{C}^{\mathrm{T}}\right)^{+} \mathrm{D}_{\mathrm{C}}{ }^{2} \operatorname{diag}\left(\mathrm{a}_{(i)}\right)\right] \\
& \times\left[\sum_{k=1}^{K}\left(\mathbf{D}_{\mathrm{A}} \operatorname{diag}\left(\mathrm{c}_{(k)}\right)\right)^{2}+\sum_{i=1}^{I}\left(\mathrm{D}_{\mathrm{C}} \operatorname{diag}\left(\mathrm{a}_{(i)}\right)\right)^{2}\right]^{+} .
\end{aligned}
$$

利用交替归一加权残差法的迭代步骤和校正的 过程与 ATLD算法相似, 最终计算得到相对激发矩阵 A, 相对发射矩阵 B 和相对浓度矩阵 C. 


\section{4 核一致诊断法(CORCONDIA)}

尽管本文所采用的这两种二阶校正算法对组分数 不敏感, 即只要求用于拟合三维成分模型的因子数 不小于真实的组分数即可, 但是根据实际应用经验, 如果拟合模型的因子数过大时会导致模型误差增加, 使校正结果与真实值之间的偏差增大. 因此, 在利用 二阶校正算法解析数据之前，为了保证预测结果的 准确性，有必要对组分数进行预估计. 核一致诊断法 (CORCONDIA) 是由Bro等人 ${ }^{[16]}$ 提出的确定组分数的 方法. 该方法通过计算平行因子分析(PARAFAC)模 型中超对角阵 $\underline{\mathbf{T}}$ 和最小二乘拟合阵 $\mathbf{G} 之$ 间的相似程度, 即核一致值(core-consistency)来估计组分数:

$$
\text { core-consistency }=100 \times\left[1-\frac{\sum_{d=1}^{F} \sum_{e=1}^{F} \sum_{f=1}^{F}\left(g_{d e f}-t_{d e f}\right)^{2}}{\sum_{d=1}^{F} \sum_{e=1}^{F} \sum_{f=1}^{F} t_{d e f}^{2}}\right],
$$

$F$ 是模型的因子数; $g_{d e f}$ 为立方阵 $\mathbf{G}$ 的元素; $t_{d e f}$ 是立方 阵 $\mathbf{T}$ 的元素. 对于理想的 PARAFAC 模型, 超对角阵 $\underline{\mathbf{T}}$ 和最小二乘拟合阵 $\mathbf{G}$ 应该非常相似，此时的核一致 值将会等于 $100 \%$. 通常，当核一致值大于或等于 $60 \%$ 时，模型也被认为接近三线性，但当核一致值低 于 $60 \%$ 时，模型则被认为偏离三线性. 所以根据核一 致值的变化情况可以获得准确的组分数.

\section{5 品质因子}

分析品质因子，例如灵敏度(SEN)、选择性(SEL) 和检测下限(LOD), 通常用来验证预测结果的准确性 或被选作算法比较. 在二阶校正中，分析品质因子的 估计跟纯分析信号的计算密切相关, SEN 是指单位浓 度的纯分析信号，而 SEL 是指灵敏度和总信号的比 值. 本文根据文献[17]所报道的公式来计算 SEN 和 SEL:

$$
\begin{aligned}
& \mathrm{SEN}=k\left\{\left[\left(\mathrm{~A}^{\mathrm{T}} \mathrm{A}\right)^{-1}\right]^{*}\left[\left(\mathrm{~B}^{\mathrm{T}} \mathrm{B}\right)^{-1}\right]\right\}_{n n}{ }^{-1 / 2}, \\
& \mathrm{SEL}=\left\{\left[\left(\mathrm{A}^{\mathrm{T}} \mathrm{A}\right)^{-1}\right]^{*}\left[\left(\mathrm{~B}^{\mathrm{T}} \mathrm{B}\right)^{-1}\right]\right\}_{n n}{ }^{-1 / 2},
\end{aligned}
$$

其中下标 $n n$ 代表矩阵的第 $(n, n)$ 个元素; $k$ 为组分 $n$ 在 单位浓度时的总信号, 它也是浓度得分参数. 符号* 表示 Hadamard 乘积.

根据下式 ${ }^{[18,19]}$ 估计检测下限(LOD):

$$
\mathrm{LOD}=3.3 s(0) \text {, }
$$

其中 $s(0)$ 为 3 个背景空白样本的预测浓度偏差.

\section{3 实验部分}

\section{1 试剂}

噻苯咪唑购自 Sigma 公司, 乙酸乙酯(天津市大 茂化学试剂厂)、无水 $\mathrm{MgSO}_{4}$ 和 $\mathrm{NaCl}$ 都是分析纯, 桔 子为市售.

\section{2 主要仪器}

日立 F-4500 型荧光分光光度计(日本日立公司), SIGMA3K30 型高速冷冻离心机(德国 Sigma 公司), KQ-250TDB 型高频数控超声清洗器(昆山市超声仪 器有限公司), 所有程序在 Matlab 的环境下运行.

\section{3 实验方法}

用乙酸乙酯配成 $30 \mu \mathrm{g} \cdot \mathrm{mL}^{-1}$ 的噻苯咪唑储备液, 储存于冰箱中, 实验当天, 再用乙酸乙酯将母液稀释 成浓度为 $0.3 \mu \mathrm{g} \cdot \mathrm{mL}^{-1}$ 工作液. 桔子剥皮后捣烂，称 取桔子混合物 $15.0 \mathrm{~g}$, 称取 $5.9 \mathrm{~g}$ 无水 $\mathrm{MgSO}_{4} 、 2.5 \mathrm{~g}$ $\mathrm{NaCl}$ 与其混合 $5 \mathrm{~min}$, 再加入 $50 \mathrm{~mL}$ 的乙酸乙酯超声 $10 \mathrm{~min}$, 然后再离心 $15 \mathrm{~min}\left(4200 \mathrm{r} \cdot \mathrm{min}^{-1}\right)$, 取上层清 液, 用于回收实验. 所有实验样本都是根据噻苯咪唑 的线性范围以及桔子中可能存在的噻苯咪唑的浓 度 ${ }^{[11]}$ 来配制的。

校正样的配制: 在 8 个 $10 \mathrm{~mL}$ 容量瓶中分别加入 不同体积的工作液,用乙酸乙酯定容，得到浓度分别 为 $3 、 6 、 9 、 12 、 15 、 18 、 21 、 24 \mathrm{ng} \cdot \mathrm{mL}^{-1}$.

实际样的配制：在 6 个 $10 \mathrm{~mL}$ 容量瓶中分别加入 不同量的工作液，同时添加 $200 \mu \mathrm{L}$ 的桔子提取液(浓 度见表 1).

表 1 ATLD/ANWE 算法分别与三维荧光相结合测定桔子 提取液中 $\operatorname{TBZ}(N=2)$

\begin{tabular}{rccccccc}
\hline \multirow{2}{*}{ 样本 } & 实际浓度 & \multicolumn{2}{c}{ 预测浓度 $/ \mathrm{ng} \cdot \mathrm{mL}^{-1}$} & & \multicolumn{2}{c}{ 回收率 $(\%)$} \\
\cline { 7 - 7 } \cline { 6 - 7 } & $\mathrm{ng} \cdot \mathrm{mL}^{-1}$ & ATLD & ANWE & & ATLD & ANWE \\
\hline 9 & 10.5 & 10.9 & 11.6 & & 103.8 & 110.5 \\
10 & 13.5 & 13.4 & 14.4 & & 99.3 & 106.7 \\
11 & 16.5 & 16.2 & 17.4 & & 98.2 & 105.4 \\
12 & 19.5 & 20.0 & 19.9 & & 102.6 & 102.1 \\
13 & 22.5 & 20.6 & 22.3 & & 91.6 & 99.1 \\
14 & 25.5 & 26.1 & 24.7 & & 102.4 & 96.9 \\
& & & & & $99.7 \pm 3.3^{\mathrm{a})}$ & $103.5 \pm 4.1^{\mathrm{a})}$ \\
\hline
\end{tabular}

a) 平均回收率 $(\%)$ 
在对样本进行三维荧光光谱扫描过程中, 分别在 扫描前、中、后测定三个乙酸乙酯空白样本, 通过扣 除空白样本的平均值, 可以尽可能地减少溶剂引起的 拉曼散射的影响. 另外, 平行测定了 3 个桔子提取液 的空白样本，用于计算背景预测浓度的标准偏差 $s(0)$.

\section{4 参数设置}

为了避免瑞利散射峰的影响, 本文选取激发波 长范围为 272 304 nm, 每 $4 \mathrm{~nm}$ 取一个数据; 发射波 长范围为 324 384 nm, 每 $4 \mathrm{~nm}$ 取一个数据; 狭缝宽 度为 $5.0 / 5.0 \mathrm{~nm}$; 扫描速度为 $1200 \mathrm{~nm} \cdot \mathrm{min}^{-1}$. 荧光响 应数据构成了大小为 $9 \times 16 \times 14$ 的三维数据阵, 其中 9 代表激发波长数, 16 代表发射波长数, 14 代表样本 数(包括 8 个校正样和 6 个实际样).

\section{4 结果与讨论}

\subsection{TBZ 的苂光光谱}

在对噻苯咪唑进行浓度预测之前, 首先利用核 一致诊断法(CORCONDIA)对所得到的三维数阵进行 秩估计, 得到结果如图 1 所示. 在因子数小于或等于 2 时，核一致值等于 1 ; 而当因子数超过 2 时，核一致 值迅速降低到零。这样的现象说明在这个体系中, 模 型选取组分数为 2 时最接近三线性. 因此, 本文所选 组分数为 2 .

利用 ATLD 算法 $(N=2)$ 和 ANWE 算法 $(N=2)$ 分 别对苂光光谱数据进行解析, 得到的光谱图分别为 图 2 和图 3. 由图可知，两种二阶校正算法分辨得到

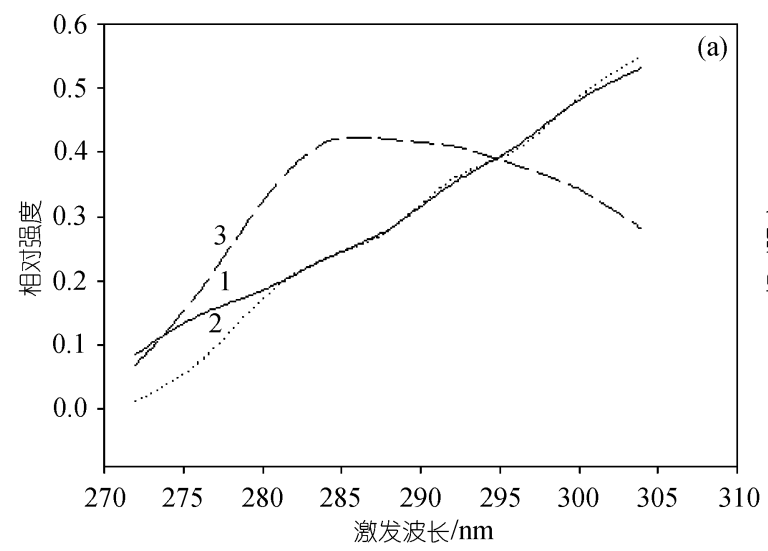

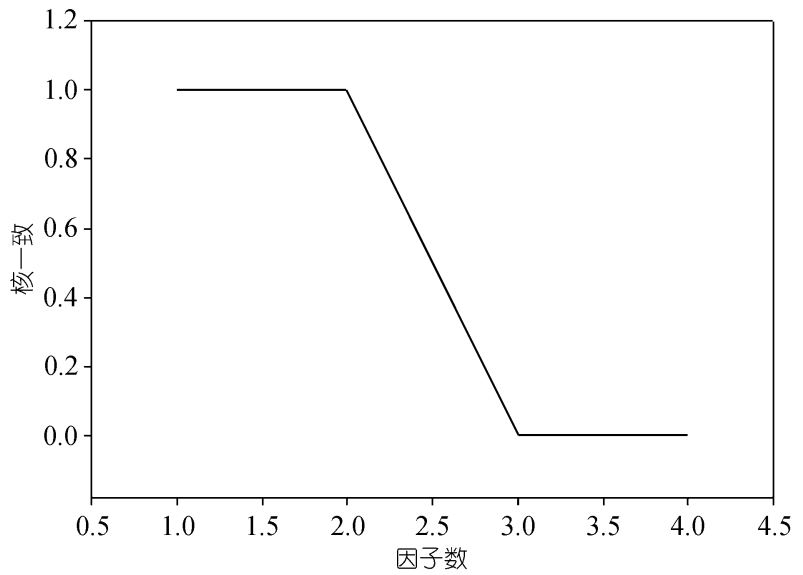

图 1 利用核一致法预估计三维数据阵的秩

的 TBZ 的激发光谱和发射光谱图十分相似，且与真 实光谱几乎完全吻合, 说明两种算法模型获得的解 是可靠的，两种方法均能对桔子提取液中的 TBZ 进 行准确分辨. 其中 $\mathrm{TBZ}$ 的最大激发波长和发射波长 分别为 $304 \mathrm{~nm}$ 和 $344 \mathrm{~nm}$.

\section{2 实际样品中 $\mathrm{TBZ}$ 含量的测定}

由图 2 和 3 可知，桔子提取液中的内源物质背景 光谱与待测组分 $\mathrm{TBZ}$ 的荧光光谱严重重叠, 会给 TBZ 的直接定量测定带来系统误差. 因此, 仅用一般 方法不经过化学分离就直接对样品进行定量分析几 乎是不可能的，而具有“二阶优势”的化学计量学方法 与三维荧光分析相结合，用 “数学分离”代替繁琐的 “化学分离”, 则可以很好地在有未知干扰存在下对感 兴趣成分进行定量分析. ATLD 和 ANWE两种算法解

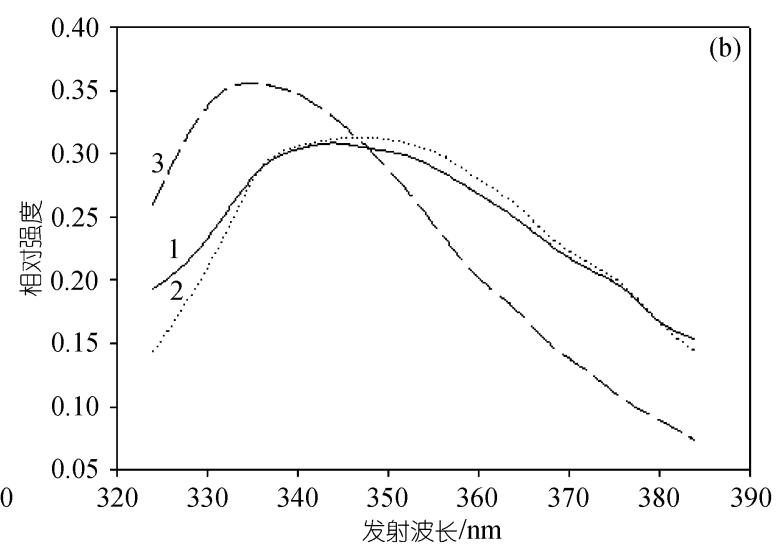

图 2 TBZ 的真实光谱和利用 ATLD 分辨得到的光谱 $(N=2)$

(a) 激发光谱; (b) 发射光谱. 其中: 1. 真实的; 2 . 分辨得到的; 3 . 分辨得到的背景 

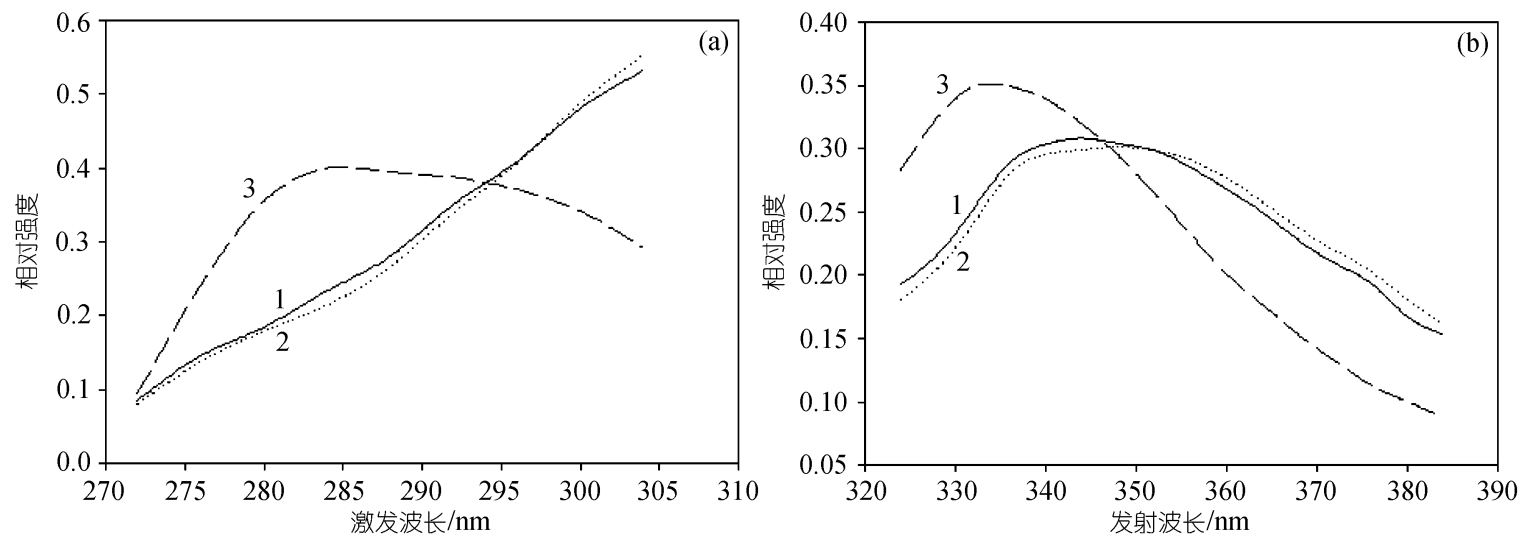

图 3 TBZ 的真实光谱和利用 ANWE 分辨得到的光谱 $(N=2)$

(a) 激发光谱; (b) 发射光谱. 其中: 1. 真实的; 2 . 分辨得到的; 3 . 分辨得到的背景

析所得结果见表 1. 两种算法解析所得校正集中 TBZ 的相对浓度与实际浓度的相关系数分别为 0.9978 和 0.9999 , 预测样中 TBZ 的平均回收率分别为 $(99.7 \pm$ $3.3) \%$ 和 $(103.5 \pm 4.1) \%$. 两算法所得预测结果都很理 想，均能实现桔子提取液中 $\mathrm{TBZ}$ 的直接快速测定.

\section{3 品质因子和椭圆置信区间}

表 2 列出了两种方法的品质因子，包括灵敏度 $(\mathrm{SEN})$ 、选择性(SEL)和检测下限(LOD). 从表 2 可以 看出, ATLD和ANWE的性能都很好, 且ATLD的品质 因子大于 ANWE. 所提供LOD的值为 3 个桔子提取液 样本的平均值. 为进一步调查所用算法的准确性, 对 实际浓度与预测浓度进行了线性回归分析 [20]，基于 椭圆置信区间测试 ${ }^{[21]}$ ，将计算得到的截距和斜率与 理想值 0 和 1 进行了比较. 图 4 绘出了ATLD和ANWE 算法的椭圆置信区间(EJCR)，结果表明标明*的理想 点 $(0,1)$ 处在EJCR中, 且用ANWE获得的椭圆小于 ATLD所获得的椭圆。这些因子进一步证明两

表 2 用 ATLD 算法和 ANWE 算法获得的桔子提取液中的 品质因子

\begin{tabular}{ccc}
\hline 品质因子 & ATLD & ANWE \\
\hline $\mathrm{SEN} / \mathrm{mL} \cdot \mathrm{ng}^{-1}$ & 2.0398 & 2.0252 \\
$\mathrm{SEL}$ & 0.1334 & 0.1286 \\
$\mathrm{LOD} / \mathrm{ng} \cdot \mathrm{mL}^{-1}$ & 2.0031 & 0.8966 \\
\hline
\end{tabular}

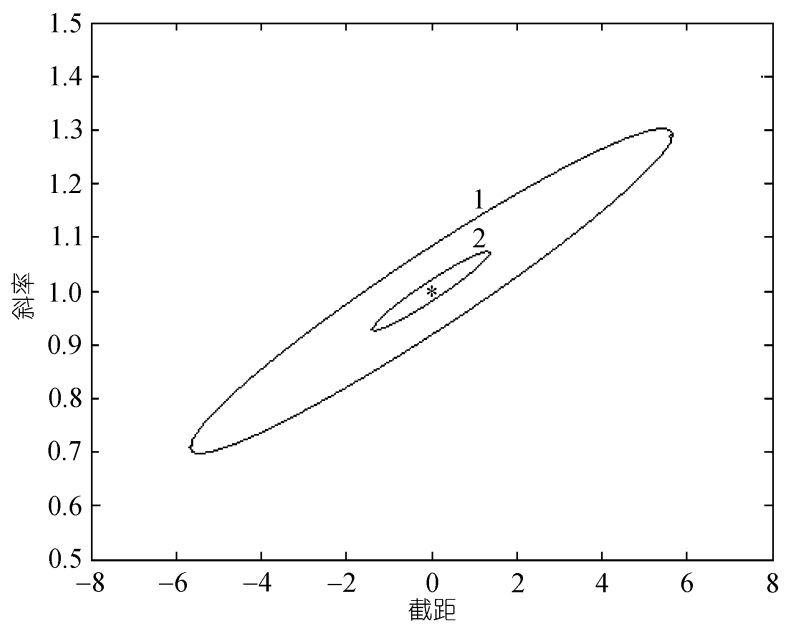

图 4 两种二阶校正方法的椭圆置信区间 1. ATLD; 2. ANWE

者算法都能给出准确的结果, 且在这个体系中 ANWE 比 ATLD 表现稍优.

\section{5 结论}

本文研究表明，交替三线性分解(ATLD)和交替 归一加权残差(AWNE)两种二阶校正算法分别与三维 荧光光谱相结合, 均能对桔子中的噻苯咪唑进行分 辨和直接快速定量测定. 品质因子和置信区间还证 明两者算法给出的结果都准确，且在这个体系中 ANWE 比 ATLD 表现稍优. 
1 Groton J P, Butler W, Feron V J, Kozianowski G, Renwick A G, Walker R. An analysis of the possibility for health implications of joint actions and interactions between food additives. Regul Toxicol Pharmacol, 2000, 31: 77-91 [DOI]

2 Vanden Heuvel William J A, Wood J S, DiGiovanni M, Walker R W. Gas-liquid chromatographic/mass spectrometric confimatory assay for thiabendazole and 5-hydroxythiabendazole. J Agric Food Chem, 1977, 25(2): 386-389 [DOI]

3 Ito Y, Ikai Y, Oka H, Hayakawa J, Kagami T. Application of ion-exchange cartridge clean-up in food analysis I. Simultaneous determination of thiabendazole and imazalil in citrus fruit and banana using high-performance liquid chromatography with ultraviolet detection. J Chromatogr A, 1998, 810: 81-87 $\underline{\text { [DOI] }}$

4 Pico-Y, Blasco C, Font G. Environmental and food applications of LC-tandem mass spectrometry in pesticide-residue analysis an overview. Mass Spectrom Rev, 2004, 23(1): 45-85[DOI]

5 Fernández-Alba A R, Tejedor A, Agüera A, Contreras M, Garrido J. Determination of imidacloprid and benzimidazole residues in fruits and vegetables by liquid chromatography-mass spectrometry after ethyl acetate multiresidue extraction. J AOAC Int, 2000, 83(3): 748-755

6 Agüera A, López S, Fernández-Alba A R, Contreras M, Crespo J, Piedra L. One-year routine application of a new method based on liquid chromatography-tandem mass spetrometry to the analysis of 16 multiclass in vegetable samples. J Chromatogr A, 2004, 1045: $125-135 \underline{[\mathrm{DOI}]}$

7 Rodríguez R, Picó Y, Font G, Maňes J. Analysis of post-harvest fungcides by micellar electrokinetic chromatography. J Chromatogr A, 2001, 924: 387-396[DOI]

8 王盛良, 黄薇. 草莓中噻苯咪唑的薄层层析检测。中国卫生检验杂志, 2000, 10(4): 426-427

9 安捷伦科技有限公司. 液相/电喷雾飞行时间质谱和液相/电喷雾离子吽质谱测定水果和蔬菜中的杀真菌剂. 环境化学, 2005, 24(4): 486-490

10 艾尔肯・依不拉音, 李明, 李兴, 沈静, 陈坚.乌鲁木齐市场水果、蔬菜中防腐剂联苯、邻苯基苯酚和噻苯咪唑的残留分析. 食品与发酵工业, 2007, 33(2): 128-132

11 García-Reyes J F, Llorent-Martínez E J, Ortega-Barrales P, Molina-Díaz A. Determination of thiabendazole residues in citrus fruits using a multicommuted fluorescence-based optosensor. Anal Chim Acta, 2006, 557: 95-100[DOI]

12 韩会新, 常风启, 陈桂茹. 蒜苔中噻苯咪唑的荧光分光光度法测定. 中国卫生检验杂志, 1998, 8(6): 340一-341

13 Rodríguez-Cuesta M J, Boqué R, Rius F X, Picón Zamora D, Martínez Galera M, GarridoFrenich A. Determination of carbendazim, fuberidazole and thiabendazole by three-dimensional excitation-emission matrix fluorescence and parallel factor analysis. Anal Chim Acta, 2003, 491: 47-56 $\underline{\text { [DOI] }}$

14 Wu H L, Shibukawa M, Oguma K. An alternating trilinear decomposition algorithm with application to calibration of HPLC-DAD for simultaneous determination of overlapped chlorinated aromatic hydrocarbons. J Chemom, 1998, 12(1): 1-26[DOI]

15 Xia A L, Wu H L, Zhu S H, Han Q J, Zhang Y, Yu R Q. Determination of psoralen in human plasma by using excitation-emission matrix fluorescence coupled to second-order calibration. Anal Sci, 2008, in press

16 Bro R, Kiers H A L. A new efficient method for determining the number of components in PARAFAC models. J Chemom, 2003, 17(5): 274-286[DOI]

17 Damiani P C, Nepote A J, Bearzotti M, Olivieri A C. A test field for the second-order advantage in bilinear least-squares and parallel factor analyses fluorescence determination of ciprofloxacin in human urine. Anal Chem, 2004, 76: 2798 - 2806 [DOI]

18 Olivieri A C, Faber N M. Standard error of prediction in parallel factor analysis of three-way data. Chemom Intell Lab Syst, 2004, 70: $75-82[\mathrm{DOI}]$

19 Faber K, Kowalski B R. Net analyte signal calculation in multivariate calibration. Anal Chem, 1997, 69: 1620-1626[DOI]

20 Arancibia J A, Escandar G M. Two different strategies for the fluorimetric determination of piroxicam in serum. Talanta, 2003, 60: $1113-1121 \underline{[\mathrm{DOI}]}$

21 González A G, Herrador M A, Asuero A G. Intra-laboratory testing of method accuracy from recovery assays. Talanta, 1999, 48: 729$736 \underline{[\mathrm{DOI}]}$ 